\title{
What Is the Next in Developing Model to Predict Survival Outcomes of Resected Pancreatic Cancer?
}

\author{
Chang Moo Kang \\ Division of HBP Surgery, Department of Surgery, Yonsei University College of Medicine, and Pancreatobiliary Cancer Center, Yonsei \\ Cancer Center, Severance Hospital, Seoul, Korea
}

\author{
Corresponding Author \\ Chang Moo Kang \\ ORCID https://orcid.org/0000-0002-5382-4658 \\ E-mail cmkang@yuhs.ac
}

\begin{abstract}
See "Development and External Validation of Survival Prediction Model for Pancreatic Cancer Using Two Nationwide Databases: Surveillance, Epidemiology and End Results (SEER) and Korea Tumor Registry System-Biliary Pancreas (KOTUS-BP)." by Jae Seung Kang, et al. on page 912, Vol. 15, No. 6, 2021
\end{abstract}

How reliably can we predict survival of patients with resected pancreatic cancer? Simply, in terms of the level of scientific evidence, single expert' opinion ranks very low. However, it is generally expected that the more clinical experiences, the more reliable the accuracy and safety in diagnosis and treatment could be obtained. It is also true that statistical analysis based on a large number of data will be able to provide the insight into prediction of prognosis and treatment strategy in our clinical practice. Kang et al. ${ }^{1}$ successfully developed survival-predicting model for pancreatic cancer by using two large scaled nationwide databases: Surveillance, Epidemiology and End Results (SEER) and Korea Tumor Registry System-Biliary Pancreas (KOTUS-BP).

SEER Program of the National Cancer Institute is an authoritative source of information on cancer incidence and survival in the United States. It routinely collects data on patient demographics, primary tumor site, tumor morphology and stage at diagnosis, first course of treatment, and follow-up for vital status since January 1973, covering approximately half of U.S. populations. ${ }^{2}$ Similarly, the Korean Association of Hepato-Biliary-Pancreatic Surgery (KAHBP) established KOTUS-BP database and actively registers the clinicopathological characteristics of the pancreatobiliary cancer patients who underwent surgery. It currently contains more than 10,000 cases of resected pancreatic cancers and biliary cancers with recent survival information. ${ }^{3}$ Therefore, as authors suggested, the present approach is believed to provide the reliable information to the patients and their family members about prognosis and postoperative individualized treatment plan on resected pancreatic cancer.

According to the present study, developed survivalpredicting model with SEER database by using age, sex, differentiation, T-stage, $\mathrm{N}$-stage, and postoperative adjuvant chemotherapy demonstrated the reliable performance (Cindex of 0.628 ) in the external validation with nationwide database in Korea (KOTUS-BP), which was also shown to be comparable to several other studies with independent model development and external validation (0.58 to 0.65$).{ }^{1}$ Then, following question seems to be much more desperate in this moment when the survival of the resected pancreatic cancer is still area of unmet needs in clinical oncology of pancreatic cancer; what can we do by using these kinds of reliable survival-predicting models in resected pancreatic cancer?

Unfortunately, most powerful variables used in the present studies (e.g., T-stage, $\mathrm{N}$-stage, differentiation, lymph node [LN] ratio, extent of LN clearance, and margin status) are mostly based on pathological examination of resected pancreatic cancer. Considering margin-negative surgical resection followed by postoperative adjuvant chemotherapy is standard treatment in resected pancreatic cancer, it might not be able to change our clinical practice to improve survival of resected pancreatic cancer if we only stick to nomogram based on pathological characteristics of resected pancreatic cancer.

Recently, more potent and effective chemotherapeutic agents against pancreatic cancer demonstrated improved survival of pancreatic cancer. In addition, evidences show- 
ing potential role of neoadjuvant chemotherapy is increasing in borderline and locally advanced pancreatic cancer. Although surgical extirpation is essential for cure of pancreatic cancer, it is admitted that many patients with resected pancreatic cancer showed early recurrence with worse survival outcomes, suggesting all resectable pancreatic cancer may not be in deed resectable. Therefore, preoperative detectable clinical parameters-based nomogram to predict survival of resected pancreatic cancer is much more useful to tailor treatment strategy and finally to improve survival outcomes of resected pancreatic cancer.

Information of preoperative detectable parameters can be measured from clinical manifestation, blood laboratory test, and preoperative image features. For example, in present study, age, sex, and tumor location are definitely preoperative detectable parameters. According to American Joint Committee on Cancer 8th cancer staging manual, T-stage is determined by tumor size, which can be measured by preoperative radiological image. $\mathrm{N}$-stage is still well-known prognostic factor in resected pancreatic cancer, but accuracy to predict $\mathrm{LN}$ metastasis by preoperative image modality is limited. However, recently, not only traditional preoperative computed tomography scan and positron emission tomography-computed tomography scan, but also noninvasive radiomics signatures extracted from preoperative contrast-enhanced computed tomography imaging showed better performance for LN metastasis prediction than traditional approaches in pancreatic cancer, suggesting potential room to guide decision making in treating pancreatic cancer. ${ }^{4-9}$ In addition, tumor marker, such as carbohydrate antigen 19-9 is known to be the most useful biomarker to predict survival of the pancreatic cancer. ${ }^{10}$

In near future, it is hoped that medical big data based on preoperative detectable parameters, the development of computational technologies, digitally transformed variables in the field of healthcare, and even genetic information from preoperative biopsy will enable researchers and practitioners to predict the prognosis and establish tailored management plan according to individualized clinical characteristics of the patients, finally leading to improving survival outcomes of resected pancreatic cancer. From that point of view, authors nicely proposed international collaborative prospective studies to develop and validate the global predictive new prediction model with preoperative variables. This will be the next in developing model to predict survival outcomes of resected pancreatic cancer for our patients.

\section{CONFLICTS OF INTEREST}

No potential conflict of interest relevant to this article was reported.

\section{ORCID}

Chang Moo Kang https://orcid.org/0000-0002-5382-4658

\section{REFERENCES}

1. Kang JS, Mok L, Heo JS, et al. Development and external validation of survival prediction model for pancreatic cancer using two nationwide databases: Surveillance, Epidemiology and End Results (SEER) and Korea Tumor Registry SystemBiliary Pancreas (KOTUS-BP). Gut Liver 2021;15:912-921.

2. National Cancer Institute Surveillance, Epidemiology and End Results Program. About the SEER program [Internet]. Bethesda: National Cancer Institute; c2021 [cited 2021 Oct 10]. Available from: https://seer.cancer.gov/about/.

3. The Korean Association of HBP Surgery. Korea Tumor Registration Database-BP [Internet]. Seoul: The Korean Association of HBP Surgery; c2021 [cited 2021 Oct 10]. Available from: https://khbp.medicaldb.co.kr.

4. Liang X, Cai W, Liu X, Jin M, Ruan L, Yan S. A radiomics model that predicts lymph node status in pancreatic cancer to guide clinical decision making: a retrospective study. J Cancer 2021;12:6050-6057.

5. Li K, Yao Q, Xiao J, et al. Contrast-enhanced CT radiomics for predicting lymph node metastasis in pancreatic ductal adenocarcinoma: a pilot study. Cancer Imaging 2020;20:12.

6. Xing H, Hao Z, Zhu W, et al. Preoperative prediction of pathological grade in pancreatic ductal adenocarcinoma based on 18F-FDG PET/CT radiomics. EJNMMI Res 2021;11:19.

7. Chang N, Cui L, Luo Y, Chang Z, Yu B, Liu Z. Development and multicenter validation of a CT-based radiomics signature for discriminating histological grades of pancreatic ductal adenocarcinoma. Quant Imaging Med Surg 2020;10:692702.

8. Kaissis G, Ziegelmayer S, Lohöfer F, et al. A machine learning algorithm predicts molecular subtypes in pancreatic ductal adenocarcinoma with differential response to gemcitabine-based versus FOLFIRINOX chemotherapy. PLoS One 2019;14:e0218642.

9. Nasief H, Zheng C, Schott D, et al. A machine learning based delta-radiomics process for early prediction of treatment response of pancreatic cancer. NPJ Precis Oncol 2019;3:25.

10. Bergquist JR, Puig CA, Shubert CR, et al. Carbohydrate antigen 19-9 elevation in anatomically resectable, early stage pancreatic cancer is independently associated with decreased overall survival and an indication for neoadjuvant therapy: a national cancer database study. J Am Coll Surg 2016;223:52-65. 